\title{
Global Phase Space of Coherence and Entanglement in a Double-Well BEC
}

\author{
Holger Hennig ${ }^{1}$, Dirk Witthaut ${ }^{2}$, David K. Campbell ${ }^{3}$ \\ ${ }^{1}$ Department of Physics, Harvard University, Cambridge, MA 02138, USA \\ ${ }^{2}$ Max Planck Institute for Dynamics and Self-Organization, 37073 Göttingen, Germany and \\ ${ }^{3}$ Department of Physics, Boston University, Boston, MA 02215, USA
}

(Dated: September 22, 2018)

\begin{abstract}
Ultracold atoms provide an ideal system for the realization of quantum technologies, but also for the study of fundamental physical questions such as the emergence of decoherence and classicality in quantum many-body systems. Here, we study the global structure of the quantum dynamics of bosonic atoms in a double-well trap and analyze the conditions for the generation of many-particle entanglement and spin squeezing which have important applications in quantum metrology. We show how the quantum dynamics is determined by the phase space structure of the associated mean-field system and where true quantum features arise beyond this 'classical' approximation.
\end{abstract}

PACS numbers: 03.75.Gg, 03.75.Lm, 67.85.Hj

Maintaining and controlling the coherence of quantum systems over time is one of the major challenges in contemporary physics. Low-temperature quantum gases trapped in optical lattices are an important instance of this challenge, for they provide versatile testbeds both for idealized models of exotic solid state systems and for applications in quantum optics and quantum information processing [14]. A Bose-Einstein condensate (BEC) loaded in two linearly coupled wells, called a 'BEC dimer' or 'Bosonic Josephson Junction' [5] is a particularly appealing case, as its quantum behavior is both theoretically tractable [6] 9] and experimentally accessible. In particular, BEC dimers have been shown to allow coherent manipulation of quantum states even on an atom chip, enabling matter-wave interferometry [10, long phase coherence times and number squeezing [11[13, and proposed chip-based gravity detectors 14]. Related questions have been addressed in the context of the mathematically equivalent Lipkin-Meshkov-Glick model [15].

For large atom numbers, the coarse dynamics of the BEC dimer is excellently described by a 'classical' meanfield approximation. Recent experiments have precisely mapped out the emerging classical phase space structure [16, 17], showing macroscopic quantum oscillations as well as the emergence of self-trapped states through a 'classical' bifurcation. Recent theoretical studies have focused on strategies to prepare highly entangled EinsteinPodolsky-Rosen (EPR) states in the BEC dimer [18 21]. These truly quantum phenomena appear to depend on the structure of the associated classical phase space [69. 18, but what is the exact correspondence?

Here, we introduce a global phase space (GPS) picture of the dynamics of the quantum dimer, focussing on entanglement, spin squeezing and decoherence of the atoms. Our global picture shows that both the time evolution of entanglement and coherence depend very fundamentally on the initial coherent state. In particular, we find that self-trapping (ST) excellently supports many-particle entanglement. To link quantum and classical aspects of the dynamics, we approach the problem from three perspec- tives. First, for the full quantum problem, we employ the two-site Bose-Hubbard model and solve for all of the above quantum observables as functions of time. Second, we recall that the standard mean-field treatment of the dimer - the two-site Gross-Pitaevski equation - corresponds to an integrable classical dynamical system, and we discuss the nature of the trajectories in this system. Third, we use a recently developed semiclassical 'Liouville dynamics approach' (LiDA) [22, which we argue is intermediate between the fully quantum and the classical systems. Comparing these three perspectives, our GPS approach provides an intuitive understanding of the quantum observables mentioned above and insights into true quantum effects beyond the mean-field or semiclassical approaches.

Quantum and semiclassical dynamics.- The coherent dynamics of the Bose-Einstein condensate dimer is given by the two-mode Bose-Hubbard Hamiltonian (BHH)

$$
H=-J\left(\hat{a}_{1}^{\dagger} \hat{a}_{2}+\hat{a}_{2}^{\dagger} \hat{a}_{1}\right)+\frac{U}{2}\left(\hat{a}_{1}^{\dagger 2} \hat{a}_{1}^{2}+\hat{a}_{2}^{\dagger 2} \hat{a}_{2}^{2}\right)
$$

where $\hat{a}_{j}$ denotes the annihilation of one bosonic atom in state $j$. The modes can be either realized by two sites in a double-well trap [23, 24]) or two internal states of the atoms [17]. The tunneling rate $J$ and the on-site interaction $U$ can be tuned individually, e.g., via a Feshbach resonance or by changing the depth of atom trap [1, 13, 17, 25, 27]. Throughout this paper we set $\hbar=1$, thus measuring all energies in frequency units. If not stated otherwise, we assume that $J=10 \mathrm{~s}^{-1}$, which is a common setting in ongoing experiments [13, 17, 27, and we assume a total number of $N=40$ atoms.

The mean-field counterpart of the Bose-Hubbard dimer is a two-mode Gross-Pitaevskii or nonlinear Schrödinger equation. This equation can be rewritten as an integrable classical Hamiltonian system with the Hamiltonian [28]

$$
H_{c l}(z, \varphi)=\frac{\Lambda z^{2}}{2}-\sqrt{1-z^{2}} \cos (\varphi) .
$$

The mean-field (classical) phase space consists of the two 

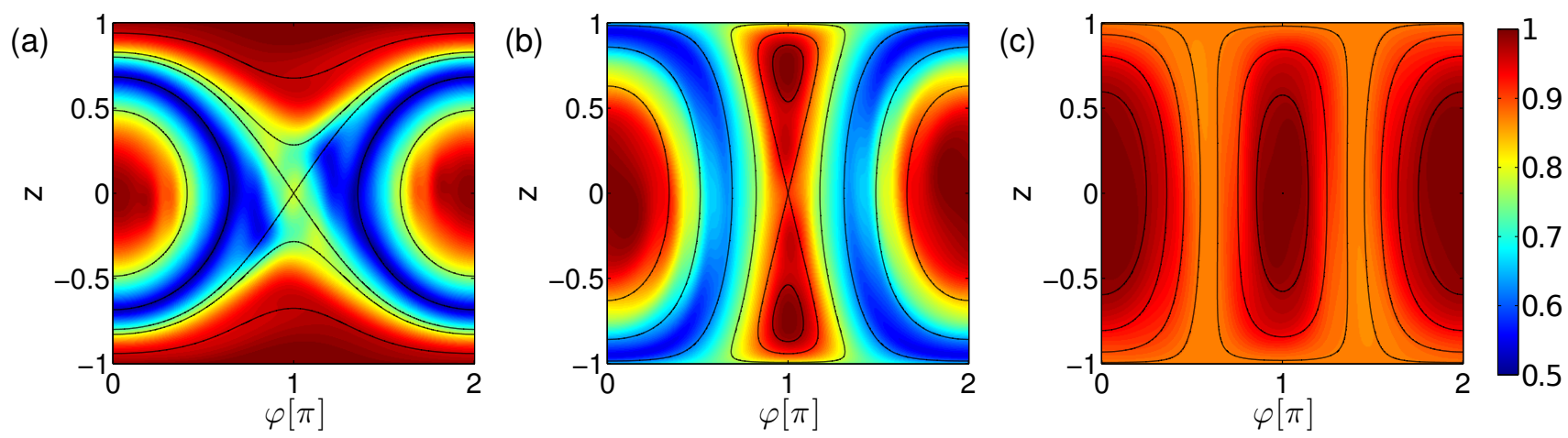

Figure 1: Global phase space structure of the Bose-Hubbard dimer for (a) $\Lambda=5$, (b) $\Lambda=1.5,(\mathbf{c}) \Lambda=0.5$. The color code shows the condensate fraction $c_{\tau}(\varphi, z)$ at $\tau=1 \mathrm{~s}$ as a function of the initial state $|\varphi, z\rangle$. Lines of equal condensate fraction mimic the classical trajectories (black lines), despite the clear qualitative differences for the three values of $\Lambda$ shown. However, we observe strong deviations in the coherence properties of the quantum state, such as S-symmetry breaking and enhanced condensate fraction near the unstable fixed point $F_{2}=(\pi, 0)$, which are clearly beyond the classical mean-field description.

conjugate variables, which following previous usage [28], we have chosen to be the relative phase $\varphi=\varphi_{1}-\varphi_{2} \in$ $[0,2 \pi)$ between the two wells and the population imbalance $z=\left(N_{1}-N_{2}\right) / N \in[0,1]$, where $N_{1}$ and $N_{2}$ denote the number of atoms in each well and $N=N_{1}+N_{2}$ is the total atomic population. The classical trajectories simply follow the lines of constant (conserved) energy $H_{c l}(z, \varphi)=$ const. They are determined by the initial position $\left(\varphi_{0}, z_{0}\right)$ in phase space and the ratio of the interaction and the tunneling energy $\Lambda=U N /(2 J)$.

The Gross-Pitaevskii equation implicitly assumes a pure BEC at all times. Some quantum features beyond this rough approximation, in particular the quantum mechanical spreading over time, can be included if a quantum state $|\Psi\rangle$ is represented by a (quantum) phasespace density such as the Husimi function $Q(\varphi, z)=$ $\left|\left\langle\varphi, z \mid \varphi_{0}, z_{0}\right\rangle\right|^{2}$ instead of a single trajectory. Here, $|\varphi, z\rangle$ denotes an atomic coherent state [29, which is nothing but a pure BEC. As shown in [22], the dynamics of the Husimi function follows a classical Liouville equation with the Hamiltonian (2) plus quantum correction terms vanishing as $1 / N$. In the semiclassical LiDA [22] we thus represent an initial state by an ensemble of trajectories, whose initial positions are distributed according to the Husimi function of the initial quantum state.

Global phase space structure.- We shall analyze the global phase space structure of the Bose-Hubbard dimer with special respect to its classical and quantum properties. Therefore, we consider the dynamics of an initially pure BEC as a function of the parameters $z_{0}$ and $\varphi_{0}$ with a focus on the purity of the condensate and the emergence of entanglement and spin-squeezing. The purity is measured by the condensate fraction $c_{\tau}$ defined as the maximum eigenvalue of the reduced single-particle density matrix (SPDM) $\rho_{i j}=\left\langle a_{i}^{\dagger} a_{j}\right\rangle / N[9,[22$, 30]. A related GPS approach was introduced in terms of the phase space entropy [31, which provides great insight into global dynamical properties of the system.

The global phase space structure of the Bose-Hubbard dimer is shown in Fig. 1, where the condensate fraction $c_{\tau}(\varphi, z)$ at time $\tau=1 \mathrm{~s}$ is plotted as a function of the initial state $\left|\varphi_{0}, z_{0}\right\rangle$ for three different values of $\Lambda$. The corresponding classical mean-field dynamics is overlaid as solid black lines. For $\Lambda<1$, the atoms show simple Rabi oscillations between the wells with stable fixed points at $F_{1}=(0,0)$ and $F_{2}=(\pi, 0)$. According to the average phase $\bar{\varphi}$, the oscillations are referred to as 'zero-phase' or plasma oscillations $(\bar{\varphi}=0)$ around $F_{1}$ and ' $\pi$-phase' oscillations $(\bar{\varphi}=\pi)$ around $F_{2}$. The classical dynamics undergoes a bifurcation at $\Lambda=1$, separating the Rabi $(0<\Lambda<1)$ and Josephson $(\Lambda>1)$ regimes. The fixed point $F_{2}$ becomes hyperbolically unstable for $\Lambda>1$, bifurcating into two self-trapping (ST) fixed points at $F_{S T}=\left(\pi, z_{S T}\right)$, where $z_{S T}= \pm \sqrt{1-1 / \Lambda^{2}}$ [28. In addition to $\pi$-phase ST with average phase $\pi$ (Fig. 1 1 b), also 'running phase' ST appears for $\Lambda>2$ (see Fig. 11a).

Comparing the condensate fraction $c_{\tau}(\varphi, z)$ with the mean-field dynamics we find that lines of equal $c_{\tau}(\varphi, z)$ mimic the classical trajectories in many respects. The GPS mapping for $c$ clearly reflects zero- and $\pi$-phase oscillations and the stable fixed points (Fig. 1(c)) as well as ST (Fig. 1(a-b)). A drastic drop in the condensate fraction is observed in particular near the classical separatrices, indicating a deviation from a pure BEC and thus the failure of a simple mean-field description. Notably, this loss of coherence is not strongest at the unstable fixed point, cf. Fig. 1 (a-b). Furthermore, the quantum dynamics breaks the symmetry $S:(\varphi, z) \rightarrow(\varphi,-z)$, as $c_{\tau}(S(\varphi, z)) \neq c_{\tau}(\varphi, z)$. In the classical limit $S$ is preserved: $H(S(\varphi, z))=H(\varphi, z)$, see Eq. 2). Of course, both the classical and the quantum dynamics are symmetric with respect to $(\varphi, z) \rightarrow(-\varphi,-z)$, which translates to relabeling wells $1 \leftrightarrow 2$ without changing the initial state.

The correspondence of quantum and classical phase space structure is more than a qualitative coincidence. In Fig. 2 (a,b) we compare the quantum results (based upon the $\mathrm{BHH}$ ) for the minimum condensate fraction with the 

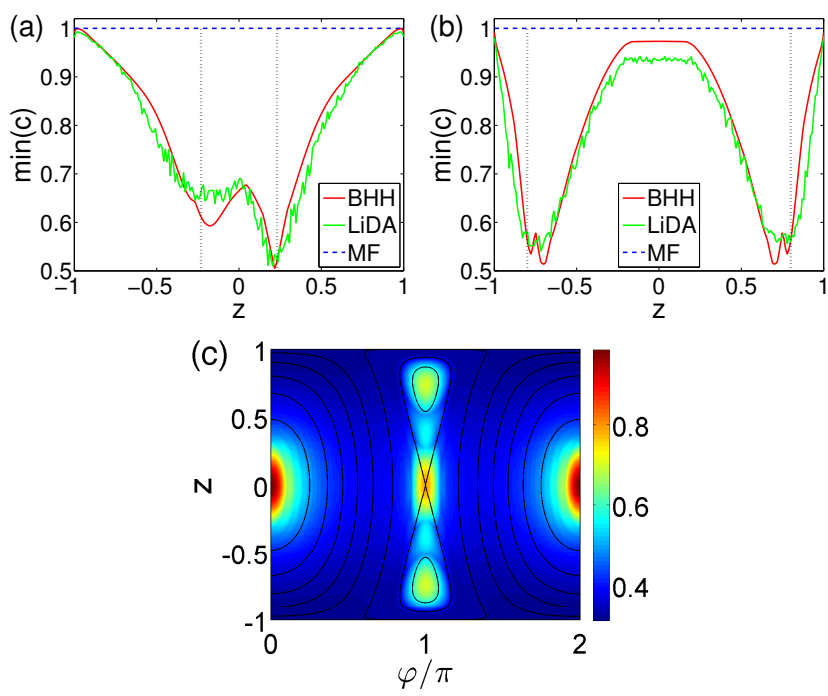

Figure 2: The GPS approach reveals novel features of quantum dynamical origin beyond the classical mean-field description and the semiclassical LiDA. (a-b) Sections through the GPS for $\Lambda=5$ comparing quantum results (based upon the BHH, red curves) with LiDA (green curves). The mean-field (MF) description assumes a pure BEC (blue dashed line). (a) Shown is the condensate fraction $\min \left(c_{\tau}(0.85 \pi, z)\right)$ in the time interval $0<\tau<0.5 \mathrm{~s}$. The point $(0.85 \pi, 0)$ is near the hyperbolic fixed point. The LiDA reproduces $S$-symmetry breaking, but can distinctly deviate from the quantum results near the hyperbolic fixed point and the separatrices (dashed vertical lines). (b) For $\min \left(c_{\tau}(0, z)\right)$ the LiDA deviates from the quantum dynamics for $|z| \lesssim 0.8$, i.e. near the separatrices (which are located at $z= \pm 0.8$, dashed lines) (c) Large condensate fraction $c_{\tau}$ in the vicinity of the classical fixed points is reproduced qualitatively by a time-independent measure. The color code shows the maximum overlap of the initial state with a stationary state, $A(\varphi, z)=\max _{n}\left|\left\langle\varphi, z \mid E_{n}\right\rangle\right|$ for $\Lambda=1.5$. However, several quantum features depicted in the GPS, such as the symmetry breaking, are not reproduced by this measure, indicating that these are of dynamical origin.

prediction of the semiclassical LiDA. The good agreement of both quantities reveals that the loss of quantum coherence $c_{\tau}(\varphi, z)$ can be mostly attributed to the classical spreading of Husimi function. Around a stable fixed point neighboring trajectories remain close for all times, such that there is no spreading.

But why does the quantum coherence remain reasonably high near the unstable fixed point? We recall that Eq. (2) can be mapped to a pendulum with variable length $l(z)=\sqrt{1-z^{2}}$ and angular velocity $z=\dot{\varphi}$. Within that picture, the unstable fixed point $F_{2}=(\pi, 0)$ is reached when the pendulum is in its upright position with no angular velocity. Close to the unstable fixed point, the classical dynamics slows down asymptotically ("freezes"); therefore spreading near the unstable fixed point is slow. Moreover, when the trajectory is moving towards $F_{2}$ the dynamics slows down, while moving away from $F_{2}$ the pendulum accelerates, which breaks the $S$-symmetry. On the contrary, close to the separatrix, the classical dynamics leads to delocalization in phase space which induces decoherence of a many particle state [22, 31].

To study to what extent these features can be captured in a time-independent framework, we analyze how much a state $|\varphi, z\rangle$ overlaps with an eigenstate $\left|E_{n}\right\rangle$ of the Bose-Hubbard dimer. Figure 2 (c) shows the maximum overlap $A_{\max }(\varphi, z)=\max _{n}\left|\left\langle\varphi, z \mid E_{n}\right\rangle\right|$ for the same parameters as in Fig. 1 (b). The overlap with the eigenfunctions is high at the stable fixed points and minimal along most parts along the separatrix. This reveals one possible mechanism to guarantee long-time coherence of a BEC: If the overlap approaches unity, the initial state is almost stationary such that the condensate fraction remains virtually constant. This is consistent with (but the converse of) the results reported in refs. [22, 31, where it was shown that delocalization in phase space induces decoherence of a quantum state. Moreover, there is as well an eigenfunction localized around the unstable fixed point $F_{2}$, which explains the surprisingly slow decoherence of the quantum state localized at this point.

Entanglement.- A distinguishing feature of experiments with two-mode BECs is that the quantum state of the atoms can be manipulated with astonishing precision. In particular, the atoms can be strongly entangled, which enables important applications in precision quantum metrology [13, 32, 33. For the important special case of EPR entanglement, a simple criterion reads $E>0$, where $E=\left|\left\langle\hat{a}_{1}^{\dagger} \hat{a}_{2}\right\rangle\right|^{2}-\left\langle\hat{a}_{1}^{\dagger} \hat{a}_{1} \hat{a}_{2}^{\dagger} \hat{a}_{2}\right\rangle$ [19, 20. Proceeding in the same way as for the condensate fraction, we obtain a GPS picture for the EPR entanglement shown in Fig. $3(\mathrm{a}, \mathrm{b})$. The measure $E_{\tau}(\varphi, z)$ again excellently mimics the classical phase space trajectories, however, an actual entangled state (i.e., $E>0$ ) is found at time $\tau=1 \mathrm{~s}$ only near the classical ST fixed points $\left(\pi, z_{S T}\right)$. Movies showing the temporal evolution of $E_{\tau}(\varphi, z)$ for $\tau \in[0,3] \mathrm{s}$ can be found in the supplementary material. Strikingly, the GPS for entanglement measured at different times $\tau$ unveils the following behavior: If entanglement appears on timescales long compared to the period of the plasma oscillations, then entanglement is concentrated around one or more fixed points in phase space, which, surprisingly, includes the unstable fixed point $F_{2}$.

Given the apparent similarity of the GPS for $E$ and the condensate fraction $c$, we ask, how is $E$ related to $c$ ? By approximating $\left\langle\hat{a}_{1}^{\dagger} \hat{a}_{1} \hat{a}_{2}^{\dagger} \hat{a}_{2}\right\rangle \approx\left\langle\hat{a}_{1}^{\dagger} \hat{a}_{1}\right\rangle\left\langle\hat{a}_{2}^{\dagger} \hat{a}_{2}\right\rangle$ we find a semiclassical measure $E_{\mathrm{sc}}=\left|\left\langle\hat{a}_{1}^{\dagger} \hat{a}_{2}\right\rangle\right|^{2}-\left\langle\hat{a}_{1}^{\dagger} \hat{a}_{1}\right\rangle\left\langle\hat{a}_{2}^{\dagger} \hat{a}_{2}\right\rangle=$ $N^{2}\left(c^{2}-c\right)$. In Fig. 3(c) a GPS for $E_{\mathrm{sc}}$ is shown for the same parameters, with remarkable qualitative agreement with the exact quantum results for $E$. In Fig. 3(d) we report the temporal evolution of $E$ and $E_{\mathrm{sc}}$ of the state $|0.2,0\rangle$ which shows surprisingly good agreement except for an offset. However, as $E_{\mathrm{sc}} \leq 0$ by definition, it does not serve as an entanglement criterion.

Spin squeezing indicates a form of many-particle entanglement which is particularly important for quantum metrology [13, 32]. A state is spin-squeezed if the 

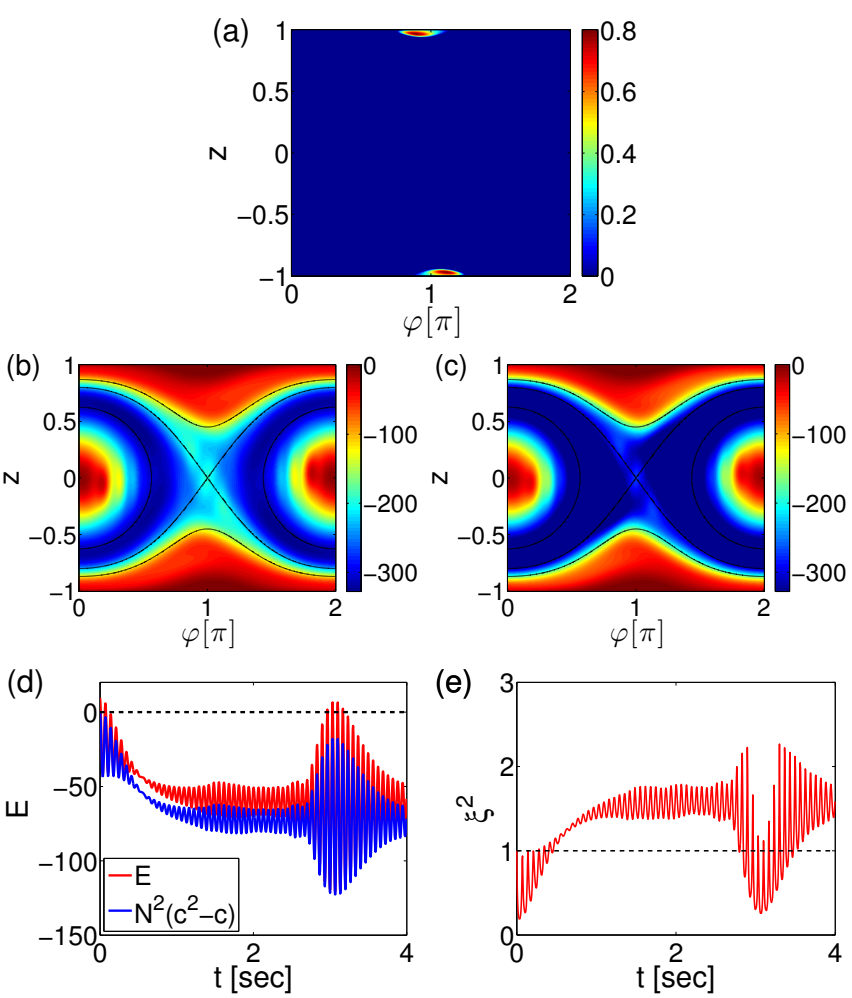

Figure 3: Global phase space structure of EPR entanglement and spin squeezing for $\Lambda=5$. (a) EPR entanglement $E>0$ is found at $\tau=1 \mathrm{~s}$ solely around the ST fixed points. (b,c) The entanglement parameter $E_{\tau}(\varphi, z)$ (b) and the semiclassical approximation $E_{s c}=N^{2}\left(c^{2}-c\right)(\mathbf{c})$ at time $\tau=1 \mathrm{~s}$ are shown as a function of the initial state $|\varphi, z\rangle$. While these snapshots depict the GPS at $\tau=1 \mathrm{~s}$, the movie in the supplementary material shows the time evolution of the GPS in timesteps of $0.025 \mathrm{~s}$. (d,e) Time evolution of EPR entanglement (d) and spin squeezing (e) for an initially pure BEC $|\varphi, z\rangle=|0,0.2\rangle$. The semiclassical approximation reproduces the overall behavior quite well but exhibits an almost constant shift. A revival of entanglement is observed at $t \approx 3 \mathrm{~s}$. Spin squeezing $\left(\xi^{2}<1\right)$ is found to be in excellent agreement with the EPR entanglement result $(E>0)$ at $t \approx 3 \mathrm{~s}$.

quantum uncertainty in a Bloch sphere representation is smaller than that of an atomic coherent state, i.e., a pure BEC. This representation is defined via the operators $\hat{J}_{x}=\frac{1}{2}\left(\hat{a}_{2}^{\dagger} \hat{a}_{1}+\hat{a}_{1}^{\dagger} \hat{a}_{2}\right), \hat{J}_{y}=\frac{i}{2}\left(\hat{a}_{2}^{\dagger} \hat{a}_{1}-\hat{a}_{1}^{\dagger} \hat{a}_{2}\right)$, and $\hat{J}_{z}=\frac{1}{2}\left(\hat{a}_{1}^{\dagger} \hat{a}_{1}-\hat{a}_{2}^{\dagger} \hat{a}_{2}\right)$, which form an angular momentum algebra with quantum number $J=N / 2$ [22, 29, 30]. A quantum state is spectroscopically squeezed if the parameter 33 .

$$
\xi^{2}:=\frac{N\left(\Delta \hat{J}_{n_{1}}\right)^{2}}{\left\langle\hat{J}_{n_{2}}\right\rangle^{2}+\left\langle\hat{J}_{n_{3}}\right\rangle^{2}}
$$

is smaller than one. Here, $\hat{J}_{n_{i}}=n_{i} \cdot \hat{J}$ is the projection of the total angular momentum operator $\hat{J}$ onto $n_{i}$, where $n_{1,2,3}$ are mutually orthogonal unit vectors and $\Delta \hat{J}_{n}$ is the variance of $\hat{J}_{n}$. In Fig. 3 (e) we report the dynamical evolution of $\xi^{2}$ for an initially pure BEC $|0.2,0\rangle$ in excellent agreement with $E(t)(\mathrm{d})$, including the entanglement revival at $t \approx 3 \mathrm{~s}$.

Discussion. - While chaotic classical dynamics has been shown typically to lead to a fast (exponential) decay of the coherence of a many-particle state [34, little is known about the coherence of quantum self-trapped states (especially in a high dimensional phase space), where the classical counterparts are (quasi-)periodic orbits, although some insight can be provided by semiclassical approaches beyond the mean-field limit [35] 37]. Fig. 2 reveals one aspect of the coherence of self-trapped states. For $z$ near \pm 1 the many-particle state is close to a pure condensate at time $\tau$, whereas the condensate fraction drops drastically well before the separatrix (dashed line) is reached. In Fig. 1(a-b) the dark red regions around the fixed points $F_{\mathrm{ST}}$ further confirm that the self-trapped regions maintain high condensate fraction. Hence, ST is a mechanism to preserve coherence of many-particle states. In contrast, in the vicinity of the separatrix the condensate fraction falls off sharply.

We have analyzed the connection between quantum observables of the BEC dimer and the structure of the underlying classical phase space, including fixed points, separatrices, and ST. The question remains: how well do our results carry over beyond the Bose-Hubbard model? Recent numerical studies beyond the Bose-Hubbard model (including higher-lying states in the individual wells) [38, 39] show that ST is only present as long as the system remains coherent. Hence, there is evidence that our results do reflect a fundamental relation between ST and coherence of Bosonic quantum systems.

In larger optical lattices, the self-trapped states of the dimer correspond to 'discrete breathers' or 'intrinsic localized modes' [40 42]). These correspond to classical trajectories which are practically embedded on a twodimensional torus in the high dimensional phase space and are thus (quasi-)periodic in time 4144. In other words, discrete breathers involve localization in phase space. Moreover, discrete breathers become attractive fixed points in presence of dissipation and have been found in a variety of physical and biochemical systems [40, 42. Just as delocalization in classical phase space leads to decoherence [22, 31, we expect that discrete breathers are candidates to support long-lived coherent and possibly entangled many-body states even in complex dissipative systems, such as biomolecular systems.

\section{Acknowledgments}

We thank Ted Pudlik for comments. HH acknowledges financial support by the Deutsche Forschungsgemeinschaft (DFG, grant no. HE 6312/1-1). DKC thanks Boston University for partial support of this research and the Kavli Institute for Theoretical Physics for its hospitality during the completion of this work. 
[1] I. Bloch, Nat. Phys. 1, 23 (2005).

[2] W. S. Bakr, J. I. Gillen, A. Peng, S. Fölling, and M. Greiner, Nature 461, 74 (2009).

[3] W. S. Bakr, A. Peng, M. E. Tai, R. Ma, J. Simon, J. I. Gillen, S. Fölling, L. Pollet, and M. Greiner, Science 329, 547 (2010).

[4] J. Simon, W. S. Bakr, R. Ma, M. E. Tai, P. M. Preiss, and M. Greiner, Nature 472, 307 (2011).

[5] J. Javanainen, Phys. Rev. Lett. 57, 3164 (1986).

[6] A. Micheli, D. Jaksch, J. Cirac, and P. Zoller, Phys. Rev. A 67, 013607 (2003).

[7] A. Polkovnikov, Phys. Rev. A 68, 033609 (2003).

[8] K. Mahmud, H. Perry, and W. Reinhardt, Phys. Rev. A 71, 023615 (2005).

[9] D. Witthaut, F. Trimborn, and S. Wimberger, Phys. Rev. Lett. 101, 200402 (2008).

[10] T. Schumm, S. Hofferberth, L. M. Andersson, S. Wildermuth, S. Groth, I. Bar-Joseph, J. Schmiedmayer, and P. Krüger, Nature Phys. 1, 57 (2005).

[11] G. B. Jo, Y. Shin, S. Will, T. Pasquini, M. Saba, W. Ketterle, D. Pritchard, M. Vengalattore, and M. Prentiss, Phys. Rev. Lett. 98, 030407 (2007).

[12] J. Estève, C. Gross, A. Weller, S. Giovanazzi, and M. K. Oberthaler, Nature 455, 1216 (2008).

[13] C. Gross, T. Zibold, E. Nicklas, J. Estève, and M. K. Oberthaler, Nature 464, 1165 (2010).

[14] B. Hall, S. Whitlock, R. Anderson, P. Hannaford, and A. Sidorov, Phys. Rev. Lett. 98, 030402 (2007).

[15] R. Orús, S. Dusuel, and J. Vidal, Phys. Rev. Lett. 101, 025701 (2008).

[16] M. Albiez, R. Gati, J. Fölling, S. Hunsmann, M. Cristiani, and M. Oberthaler, Phys. Rev. Lett. 95, 010402 (2005).

[17] T. Zibold, E. Nicklas, C. Gross, and M. Oberthaler, Phys. Rev. Lett. 105, 204101 (2010).

[18] A. Polkovnikov, S. Sachdev, and S. Girvin, Phys. Rev. A 66, 053607 (2002).

[19] M. Hillery and M. Zubairy, Phys. Rev. Lett. 96, 050503 (2006).

[20] Q. He, M. Reid, T. Vaughan, C. Gross, M. Oberthaler, and P. Drummond, Phys. Rev. Lett. 106, 120405 (2011).

[21] N. Bar-Gill, C. Gross, I. Mazets, M. Oberthaler, and G. Kurizki, Phys. Rev. Lett. 106, 120404 (2011).

[22] F. Trimborn, D. Witthaut, and H. J. Korsch, Phys. Rev. A 79, 013608 (2009).

[23] R. Gati, J. Esteve, B. Hemmerling, T. B. Ottenstein, J. Appmeier, A. Weller, and M. K. Oberthaler, New J.
Phys. 8, 189 (2006).

[24] R. Gati, B. Hemmerling, J. Fölling, M. Albiez, and M. Oberthaler, Phys. Rev. Lett. 96, 130404 (2006).

[25] S. Inouye, M. Andrews, J. Stenger, H. Miesner, D. Stamper-Kurn, and W. Ketterle, Nature 392, 151 (1998).

[26] N. Syassen, D. Bauer, M. Lettner, T. Volz, D. Dietze, J. Garcia-Ripoll, J. Cirac, G. Rempe, and S. Durr, Science 320, 1329 (2008).

[27] E. Nicklas, H. Strobel, T. Zibold, C. Gross, B. Malomed, P. Kevrekidis, and M. Oberthaler, Phys. Rev. Lett. 107, 193001 (2011).

[28] S. Raghavan, A. Smerzi, S. Fantoni, and S. R. Shenoy, Phys. Rev. A 59, 620 (1999).

[29] W. Zhang, D. Feng, and R. Gilmore, Rev. Mod. Phys. 62, 867 (1990).

[30] C. J. Pethick and H. Smith, Bose-Einstein Condensation in Dilute Gases (Cambridge University Press, Cambridge, UK, 2008).

[31] B. Mirbach and H. Korsch, Phys. Rev. Lett. 75, 362 (1995).

[32] D. Wineland, J. Bollinger, W. Itano, and D. Heinzen, Phys. Rev. A 50, 67 (1994).

[33] A. Sorensen, L. Duan, I. Cirac, and P. Zoller, Nature 409, 63 (2000).

[34] Y. Castin and R. Dum, Phys. Rev. Lett. 79, 3553 (1997).

[35] A. Vardi and J. Anglin, Phys. Rev. Lett. 86, 568 (2001).

[36] D. Witthaut, F. Trimborn, H. Hennig, G. Kordas, T. Geisel, and S. Wimberger, Phys. Rev. A 83, 063608 (2011).

[37] F. Trimborn, D. Witthaut, H. Hennig, G. Kordas, T. Geisel, and S. Wimberger, Eur. Phys. J. D 63, 63 (2011).

[38] K. Sakmann, A. I. Streltsov, O. Alon, and L. S. Cederbaum, Phys. Rev. Lett. 103, 220601 (2009).

[39] M. Trujillo-Martinez, A. Posazhennikova, and J. Kroha, Phys. Rev. Lett. 103, 105302 (2009).

[40] D. K. Campbell, S. Flach, and Y. S. Kivshar, Phys. Today 57, 43 (2004).

[41] S. Flach and C. R. Willis, Physics Reports 295, 181 (1998).

[42] S. Flach and A. V. Gorbach, Phys. Rep. 467, 1 (2008).

[43] S. Flach and C. R. Willis, Phys. Lett. A 181, 232 (1993).

[44] H. Hennig, J. Dorignac, and D. Campbell, Phys. Rev. A 82, 053604 (2010). 